\title{
A note on type 2 degenerate poly-Frobenius-Genocchi polynomials
}

\section{Waseem A. Khan}

Department of Mathematics and Natural Sciences, Prince Mohammad Bin Fahd University, P.O Box 1664, Al Khobar 31952, Kingdom of Saudi Arabia

\author{
E-mail: wkhan1@pmu.edu.sa
}

\begin{abstract}
In [18], Kim et al. introduced the degenerate poly-Bernoulli polynomials by using polyexponential function. In this paper, we study the degenerate poly-Frobenius-Genocchi polynomials, which are called the type 2 degenerate polyFrobenius-Genocchi polynomials, by means of polyexponential function. Then, we derive some useful relations and properties. We derive type 2 degenerate poly-FrobeniusGenocchi polynomials equal a linear combination of the degenerate Frobenius-Genocchi polynomials and Stirling numbers of the first kind. Furthermore, we introduce type 2 degenerate unipoly-Frobenius-Genocchi polynomials by means of unipoly function and derive explicit multifarious properties.
\end{abstract}

Keywords: polylogarithm function, Frobenius-Genocchi polynomials, degenerate poly-Frobenius-Euler polynomials.

2010 Mathematics Subject Classification: 05A19, 11B68, 11B83, 11B95.

\section{Introduction}

Recently, Kim and his research team (see [7-18]) have studied the degenerate versions of special numbers and polynomials actively. This idea provides a powerful tool to define special numbers and polynomials of their degenerate versions. We can say that the notion of degenerate version from a special class of polynomials because of their great applicability. The most important application of these polynomials is in the theory of finite differences, analytic number theory, applications in classical analysis, and statistics. Despite the applicability of special functions in classical analysis and statistics, they also arise in communications systems, quantum mechanics, nonlinear wave propagation, electric circuit theory. electromagnetic theory, etc.

As is well known, the classical Bernoulli, Euler and Genocchi polynomials are respectively, defined by (see $[4,5,6]$ )

$$
\frac{t}{e^{t}-1} e^{x t}=\sum_{j=0}^{\infty} B_{j}(x) \frac{t^{j}}{j !},|t|<2 \pi, \frac{2}{e^{t}+1} e^{x t}=\sum_{j=0}^{\infty} E_{j}(x) \frac{t^{j}}{j !},|t|<\pi
$$

and

$$
\frac{2 t}{e^{t}+1} e^{x t}=\sum_{j=0}^{\infty} G_{j}(x) \frac{t^{j}}{j !},|t|<\pi .
$$

In the case when $x=0, B_{j}=B_{j}(0), E_{j}=E_{j}(0)$ and $G_{j}=G_{j}(0)$ are respectively, called the Bernoulli, Euler and Genocchi numbers. by

For $u \in \mathbb{C}$ with $u \neq 1$, the Frobenius-Genocchi polynomials $G_{n}^{F}(x ; u)$ are defined

$$
\frac{(1-u) t}{e^{t}-u} e^{x t}=\sum_{n=0}^{\infty} G_{n}^{F}(x ; u) \frac{t^{n}}{n !},(\text { see }[18) .
$$


In the case when $x=0, G_{n}^{F}(u)=G_{n}^{F}(0 ; u)$ are called the Frobenius-Genocchi numbers.

Obviously

$$
G_{n}^{F}(x ;-1)=G_{n}(x)
$$

For $k \in \mathbb{Z}$, Kim-Kim [7] defined the modified polyexponential function, as an inverse to the polylogarithm function by

$$
\operatorname{Ei}_{k}(x)=\sum_{n=1}^{\infty} \frac{x^{n}}{n^{k}(n-1) !} .
$$

It is worthy to note that $e(x, 1 \mid k)=\frac{1}{x} \operatorname{Ei}_{k}(x)$ and $\operatorname{Ei}_{1}(x)=e^{x}-1$. by

For any non-zero $\lambda \in \mathbb{R}$ (or $\mathbb{C}$ ), the degenerate exponential function is defined

$$
e_{\lambda}^{x}(t)=(1+\lambda t)^{\frac{x}{\lambda}}, e_{\lambda}(t)=(1+\lambda t)^{\frac{1}{\lambda}}
$$

By binomial expansion, we get

$$
e_{\lambda}^{x}(t)=\sum_{n=0}^{\infty}(x)_{n, \lambda} \frac{t^{n}}{n !},(\text { see }[12,13]),
$$

where $(x)_{0, \lambda}=1,(x)_{n, \lambda}=(x-\lambda)(x-2 \lambda) \cdots(x-(n-1) \lambda),(n \geq 1)$.

Note that

$$
\lim _{\lambda \rightarrow 0} e_{\lambda}^{x}(t)=\sum_{n=0}^{\infty} x^{n} \frac{t^{n}}{n !}=e^{x t}
$$

In $[1,2]$, Carlitz introduced the degenerate Bernoulli polynomials given by

$$
\frac{t}{e_{\lambda}(t)-1} e_{\lambda}^{x}(t)=\frac{t}{(1+\lambda t)^{\frac{1}{\lambda}}-1}(1+\lambda t)^{\frac{x}{\lambda}}=\sum_{n=0}^{\infty} \beta_{n}(x ; \lambda,) \frac{t^{n}}{n !} .
$$

The case $x=0, \beta_{n}(\lambda)=\beta_{n}(0 ; \lambda)$ are called the degenerate Bernoulli numbers.

Note that

$$
\lim _{\lambda \longrightarrow 0} \beta_{n}(x ; \lambda)=B_{n}(x) .
$$

In [17], Kim et al. considered the the degenerate Genocchi polynomials given by

$$
\frac{2 z}{e_{\lambda}(z)+1} e_{\lambda}^{u}(z)=\sum_{j=0}^{\infty} G_{j, \lambda}(u) \frac{z^{j}}{j !} .
$$

In the case when $u=0, G_{j, \lambda}=G_{j, \lambda}(0)$ are called the degenerate Genocchi numbers.

Very recently, Kim et al. [18] introduced the degenerate poly-Bernoulli polynomials defined by

$$
\frac{\operatorname{Ei}_{k}(\log (1+t))}{e_{\lambda}(t)-1} e_{\lambda}^{x}(t)=\sum_{n=0}^{\infty} \beta_{n, \lambda}^{(k)}(x) \frac{t^{n}}{n !} .
$$

Here, $\beta_{n, \lambda}^{(k)}=\beta_{n, \lambda}^{(k)}(0)$ are called the degenerate poly-Bernoulli numbers.

Kim et al. [9] introduced the degenerate Frobenius-Euler polynomials are defined by means of the generating function as follows 


$$
\frac{1-u}{(1+\lambda t)^{\frac{1}{\lambda}}-u}(1+\lambda t)^{\frac{x}{\lambda}}=\sum_{n=0}^{\infty} h_{n, \lambda}(x \mid u) \frac{t^{n}}{n !}
$$

so that

$$
h_{n, \lambda}(x \mid u)=\sum_{m=0}^{n}\left(\begin{array}{l}
n \\
m
\end{array}\right) h_{m, \lambda}(u)\left(\frac{x}{\lambda}\right)_{n-m},(n \geq 0) .
$$

When $x=0, h_{n, \lambda}(u)=h_{n, \lambda}(0 \mid u)$ are called the degenerate Frobenius-Euler numbers, (see $[8,7])$.

Note that

$$
\lim _{\lambda \longrightarrow 0} h_{n, \lambda}(x \mid u)=H_{n}(x \mid u),(n \geq 0) .
$$

It is well known that the Stirling numbers of the first kind are defined by

$$
(x)_{n}=\sum_{l=0}^{n} S_{1}(n, l) x^{l},(\text { see }[6,7]),
$$

where $(x)_{0}=1$, and $(x)_{n}=x(x-1) \cdots(x-n+1),(n \geq 1)$. From (1.10), it is easily to see that

$$
\frac{1}{k !}(\log (1+t))^{k}=\sum_{n=k}^{\infty} S_{1}(n, k) \frac{t^{n}}{n !},(k \geq 0),(\text { see }[6,9,13-15]) .
$$

In the inverse expression to (1.11), the Stirling numbers of the second kind are defined by

$$
x^{n}=\sum_{l=0}^{n} S_{2}(n, l)(x)_{l},(\text { see }[1,6,7]) .
$$

From (1.12), it is easily to see that

$$
\frac{1}{k !}\left(e^{t}-1\right)^{k}=\sum_{n=l}^{\infty} S_{2}(n, l) \frac{t^{n}}{n !},(\text { see }[8,11,13,14]) .
$$

The generating function of the degenerate Stirling numbers of the second kind [13-19] are defined by

$$
\frac{1}{n !}\left(e_{\lambda}(t)-1\right)^{n}=\sum_{l=n}^{\infty} S_{2, \lambda}(l, n) \frac{t^{l}}{l !},(n \geq 0) .
$$

In this article, we consider the generating function for the type 2 degenerate poly-Frobenius-Genocchi polynomials, which are called the type 2 degenerate polyFrobenius-Genocchi polynomials, by means of polyexponential function and derive some properties of those polynomials. In the last section, we define type 2 degenerate unipoly-Frobenius-Genocchi polynomials by means of unipoly function and includes several properties.

\section{Type 2 degenerate poly-Frobenius-Genocchi polynomials}

Let $\lambda, u \in \mathbb{C}$ with $u \neq 1$ and $k \in \mathbb{Z}$, by using the polyexponential function, we consider the type 2 degenerate poly-Frobenius-Genocchi polynomials are defined by means of the following generating function

$$
\frac{\operatorname{Ei}_{k}(\log (1+(1-u) t))}{e_{\lambda}(t)-u} e_{\lambda}^{x}(t)=\sum_{n=0}^{\infty} G_{n, \lambda}^{(F, k)}(x ; u) \frac{t^{n}}{n !} .
$$


In case when $x=0$ in $(2.1), G_{n, \lambda}^{(F, k)}(u)=G_{n, \lambda}^{(F, k)}(0 ; u)$ are called the type 2 degenerate poly-Frobenius-Genocchi numbers.

For $k=1$ in $(2.1)$, we get

$$
\frac{(1-u) t}{e_{\lambda}(t)-u} e_{\lambda}^{x}(t)=\sum_{n=0}^{\infty} G_{n, \lambda}^{F}(x ; u) \frac{t^{n}}{n !},
$$

where $G_{n, \lambda}^{F}(x ; u)$ are called the degenerate Frobenius-Genocchi polynomials.

Obviously

$$
\begin{gathered}
\lim _{\lambda \longrightarrow 0}\left(\frac{\operatorname{Ei}_{k}(\log (1+(1-u) t))}{e_{\lambda}(t)-u}\right) e_{\lambda}^{x}(t)=\sum_{n=0}^{\infty} \lim _{\lambda \longrightarrow 0} G_{n, \lambda}^{(F, k)}(x ; u) \frac{t^{n}}{n !} \\
=\frac{(1-u) t}{e^{t}-u} e^{x t}=\sum_{n=0}^{\infty} G_{n}^{(F)}(x ; u) \frac{t^{n}}{n !} .
\end{gathered}
$$

Thus, by (2.1) and (2.3), we have

$$
\lim _{\lambda \longrightarrow 0} G_{n, \lambda}^{(F, k)}(x ; u)=G_{n}^{(F, k)}(x ; u),(n \geq 0)
$$

where $G_{n}^{(F, k)}(x ; u)$ are called the type 2 poly-Frobenius-Genocchi polynomials.

It is clear that

$$
\begin{gathered}
G_{n, \lambda}^{(F, 1)}(x ; u)=G_{n, \lambda}^{(F)}(x ; u), \\
G_{n, \lambda}^{(F, 1)}(x ;-1)=G_{n}(x)(\text { see }[4]), \\
\lim _{\lambda \longrightarrow 0} G_{n, \lambda}^{(F, 1)}(x ; u)=G_{n}^{(F)}(x ; u),(n \geq 0),(\text { see }[18]) .
\end{gathered}
$$

Theorem 2.1. For $n \geq 0$, we have

$$
\begin{gathered}
\sum_{l=0}^{n}\left(\begin{array}{l}
n \\
l
\end{array}\right) \sum_{m=0}^{\infty} \frac{1}{(m+1)^{k-1}} \sum_{m=l}^{\infty} S_{1}(l+1, m+1)(x)_{n-l, \lambda} \frac{(1-u)^{l+1}}{l+1} \\
=\sum_{m=0}^{n}\left(\begin{array}{c}
n \\
m
\end{array}\right) G_{n-m, \lambda}^{(F, k)}(x \mid u)(1)_{m, \lambda}-u G_{n, \lambda}^{(F, k)}(x \mid u) .
\end{gathered}
$$

Proof. From (2.1), we have

$$
\begin{aligned}
\operatorname{Ei}_{k}(\log (1 & +(1-u) t)) e_{\lambda}^{x}(t)=e_{\lambda}(t) \sum_{n=0}^{\infty} G_{n, \lambda}^{(F, k)}(x \mid u) \frac{t^{n}}{n !}-u \sum_{n=0}^{\infty} G_{n, \lambda}^{(F, k)}(x \mid u) \frac{t^{n}}{n !} \\
& =\sum_{m=0}^{\infty}(1)_{m, \lambda} \frac{t^{m}}{m !} \sum_{n=0}^{\infty} G_{n, \lambda}^{(F, k)}(x \mid u) \frac{t^{n}}{n !}-u \sum_{n=0}^{\infty} G_{n, \lambda}^{(F, k)}(x \mid u) \frac{t^{n}}{n !} \\
& =\sum_{n=0}^{\infty}\left(\sum_{m=0}^{n}\left(\begin{array}{c}
n \\
m
\end{array}\right) G_{n-m, \lambda}^{(F, k)}(x \mid u)(1)_{m, \lambda}-u G_{n, \lambda}^{(F, k)}(x \mid u)\right) \frac{t^{n}}{n !}
\end{aligned}
$$

On the other hand,

$$
\begin{gathered}
\operatorname{Ei}_{k}(\log (1+(1-u) t)) e_{\lambda}^{x}(t) \\
=\left(\sum_{n=0}^{\infty}(x)_{n, \lambda} \frac{t^{n}}{n !}\right)\left(\sum_{m=1}^{\infty} \frac{(\log (1+(1-u) t))^{m}}{(m-1) ! m^{k}}\right)
\end{gathered}
$$




$$
\begin{gathered}
=\left(\sum_{n=0}^{\infty}(x)_{n, \lambda} \frac{t^{n}}{n !}\right)\left(\sum_{m=0}^{\infty} \frac{1}{(m+1)^{k-1}} \sum_{l=m+1}^{\infty} S_{1}(l, m+1) \frac{(1-u)^{l} t^{l}}{l !}\right) \\
=\left(\sum_{n=0}^{\infty}(x)_{n, \lambda} \frac{t^{n}}{n !}\right)\left(\sum_{l=0}^{\infty} \sum_{m=0}^{\infty} \frac{1}{(m+1)^{k-1}} \sum_{m=l}^{\infty} S_{1}(l+1, m+1) \frac{(1-u)^{l+1}}{l+1} \frac{t^{l}}{l !}\right) \\
=\sum_{n=0}^{\infty}\left(\sum_{l=0}^{n}\left(\begin{array}{l}
n \\
l
\end{array}\right) \sum_{m=0}^{\infty} \frac{1}{(m+1)^{k-1}} \sum_{m=l}^{\infty} S_{1}(l+1, m+1)(x)_{n-l, \lambda} \frac{(1-u)^{l+1}}{l+1}\right) \frac{t^{n}}{n !} .
\end{gathered}
$$

Comparing the coefficients of $\frac{t^{n}}{n !}$ on both sides of equation (2.6) and (2.7), we obtain the following theorem.

Theorem 2.2. For $n \geq 0$, we have

$$
G_{n, \lambda}^{(F, k)}(x ; u)=\sum_{m=0}^{n}\left(\begin{array}{c}
n \\
m
\end{array}\right) G_{n-m, \lambda}^{(F, k)}(u)(x)_{m, \lambda} .
$$

Proof. From (2.1), we have

$$
\begin{gathered}
\sum_{n=0}^{\infty} G_{n, \lambda}^{(F, k)}(x ; u) \frac{t^{n}}{n !}=\left(\frac{\operatorname{Ei}_{k}(\log (1+(1-u) t))}{e_{\lambda}(t)-u}\right) e_{\lambda}^{x}(t) \\
=\sum_{n=0}^{\infty} G_{n, \lambda}^{(F, k)}(u) \frac{t^{n}}{n !} \sum_{m=0}^{\infty}(x)_{m, \lambda} \frac{t^{m}}{m !} \\
\text { L.H.S }=\sum_{n=0}^{\infty} \sum_{m=0}^{n}\left(\begin{array}{c}
n \\
m
\end{array}\right) G_{n-m, \lambda}^{(F, k)}(u)(x)_{m, \lambda} \frac{t^{n}}{n !} .
\end{gathered}
$$

Therefore, by (2.1) and (2.9), we require at the desired result.

Theorem 2.3. For $k \in \mathbb{Z}$ and $n \geq 0$, we have

$$
G_{n, \lambda}^{(F, k)}(x ; u)=\sum_{l=0}^{n}\left(\begin{array}{l}
n \\
l
\end{array}\right) \sum_{m=0}^{\infty} \frac{1}{(m+1)^{k}} \sum_{m=l}^{\infty} \frac{S_{1}(l+1, m+1)(1-u)^{l}}{l+1} G_{n-l, \lambda}^{F}(x ; u) .
$$

Proof. By using equations (1.11), (2.2) and (2.1), we have

$$
\begin{gathered}
\sum_{n=0}^{\infty} G_{n, \lambda}^{(F, k)}(x ; u) \frac{t^{n}}{n !}=\left(\frac{\operatorname{Ei}_{k}(\log (1+(1-u) t))}{e_{\lambda}(t)-u}\right) e_{\lambda}^{x}(t) \\
=\frac{e_{\lambda}^{x}(t)}{e_{\lambda}(t)-u} \sum_{m=1}^{\infty} \frac{(\log (1+(1-u) t))^{m}}{(m-1) ! m^{k}} \\
=\frac{e_{\lambda}^{x}(t)}{e_{\lambda}(t)-u} \sum_{m=0}^{\infty} \frac{(\log (1+(1-u) t))^{m+1}}{m !(m+1)^{k}} \\
=\frac{e_{\lambda}^{x}(t)}{e_{\lambda}(t)-u} \sum_{m=0}^{\infty} \frac{1}{(m+1)^{k}} \sum_{n=m+1}^{\infty} S_{1}(n, m+1) \frac{((1-u) t)^{n}}{n !} \\
=\frac{1-u) t}{e_{\lambda}(t)-u} e_{\lambda}^{x}(t) \sum_{m=0}^{\infty} \frac{1}{(m+1)^{k}} \sum_{m=n}^{\infty} \frac{S_{1}(n+1, m+1)(1-u)^{n}}{n+1} \frac{t^{n}}{n !} \\
=\sum_{n=0}^{\infty} G_{n, \lambda}^{F}(x ; u) \frac{t^{n}}{n !} \sum_{m=0}^{\infty} \frac{1}{(m+1)^{k}} \sum_{m=l}^{\infty} \frac{S_{1}(l+1, m+1)(1-u)^{l}}{l !} \frac{t^{l}}{l !}
\end{gathered}
$$




$$
\text { L.H.S }=\sum_{n=0}^{\infty}\left(\sum_{l=0}^{n}\left(\begin{array}{l}
n \\
l
\end{array}\right) \sum_{m=0}^{\infty} \frac{1}{(m+1)^{k}} \sum_{m=l}^{\infty} \frac{S_{1}(l+1, m+1)(1-u)^{l}}{l+1} G_{n-l, \lambda}^{F}(x ; u)\right) \frac{t^{n}}{n !}
$$

By comparing the coefficients of $\frac{t^{n}}{n !}$, we arrive at the desired result (2.10).

Corollary 2.1. For $k \in \mathbb{Z}$ and $n \geq 0$, we have

$$
G_{n, \lambda}^{(F, k)}(u)=\sum_{l=0}^{n}\left(\begin{array}{l}
n \\
l
\end{array}\right) \sum_{m=0}^{\infty} \frac{1}{(m+1)^{k}} \sum_{m=l}^{\infty} \frac{S_{1}(l+1, m+1)(1-u)^{l}}{l+1} G_{n-l, \lambda}^{F}(u) .
$$

Corollary 2.2. For $n \geq 0$, we have

$$
G_{n, \lambda}^{F}(x ; u)=\sum_{l=0}^{n}\left(\begin{array}{l}
n \\
l
\end{array}\right) \sum_{m=0}^{\infty} \sum_{m=l}^{\infty} \frac{S_{1}(l+1, m+1)(1-u)^{l}}{l+1} G_{n-l, \lambda}^{F}(x ; u) .
$$

Corollary 2.3. For $n \geq 0$, we have

$$
G_{n, \lambda}(x)=\sum_{l=0}^{n}\left(\begin{array}{l}
n \\
l
\end{array}\right) \sum_{m=0}^{\infty} \sum_{m=l}^{\infty} \frac{S_{1}(l+1, m+1) 2^{l}}{l+1} G_{n-l, \lambda}(x) .
$$

In particular,

$$
\sum_{l=0}^{n}\left(\begin{array}{l}
n \\
l
\end{array}\right) \sum_{m=0}^{\infty} \sum_{m=l}^{\infty} \frac{S_{1}(l+1, m+1) 2^{l}}{l+1} G_{n-l, \lambda}(x)=0 .
$$

It is well-known from $([3])$ that

$$
\left(\frac{t}{\log (1+t)}\right)^{r}(1+t)^{x-1}=\sum_{n=0}^{\infty} B_{n}^{(n-r+1)}(x) \frac{t^{n}}{n !},(r \in \mathbb{C}),
$$

where $B_{n}^{(r)}(x)$ are called the higher-order Bernoulli polynomials which are given by the generating function

$$
\left(\frac{t}{e^{t}-1}\right)^{r} e^{x t}=\sum_{n=0}^{\infty} B_{n}^{(r)}(x) \frac{t^{n}}{n !}
$$

Theorem 2.4. For $n \geq 0$, we have

$$
G_{n, \lambda}^{(F, 2)}(u)=\sum_{l=0}^{l}\left(\begin{array}{l}
n \\
l
\end{array}\right) \frac{(1-u)^{l} B_{l}^{l}}{l+1} G_{n-l, \lambda}^{F}(u) .
$$

Proof. Using (1.3), we first consider the following expression

$$
\begin{gathered}
\frac{d}{d x} \operatorname{Ei}_{k}(\log (1+(1-u) x))=\frac{d}{d x} \sum_{n=1}^{\infty} \frac{(\log (1+(1-u) x))^{n}}{(n+1) ! n^{k}} \\
=\frac{1-u}{(1+(1-u) x) \log (1+(1-u) x)} \sum_{n=1}^{\infty} \frac{(\log (1+(1-u) x))^{n}}{(n+1) ! n^{k-1}} \\
=\frac{1-u}{(1+(1-u) x) \log (1+(1-u) x)} E i_{k-1}(\log (1+(1-u) x) .
\end{gathered}
$$

From (2.14), $k \geq 1$, we have

$$
\sum_{n=0}^{\infty} G_{n, \lambda}^{(F, k)}(u) \frac{x^{n}}{n !}=\frac{(1-u)^{k-1}}{e_{\lambda}(x)-u} \int_{0}^{x} \frac{1}{(1+(1-u) t) \log (1+(1-u) t)}
$$




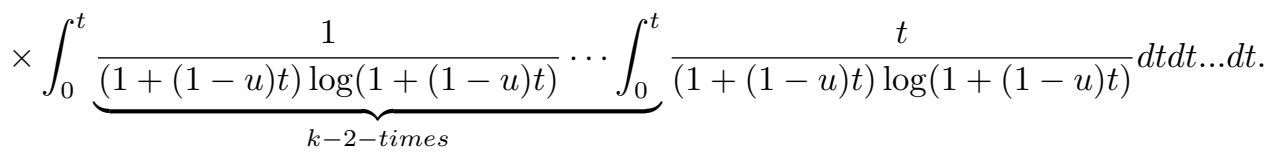

Hence, we require

$$
\begin{aligned}
\sum_{n=0}^{\infty} G_{n, \lambda}^{(F, 2)}(u) \frac{x^{n}}{n !} & =\frac{(1-u)}{e_{\lambda}(x)-u} \int_{0}^{x} \frac{(1-u) t}{(1+(1-u) t) \log (1+(1-u) t)} \\
& =\frac{(1-u)}{e_{\lambda}(x)-u} \int_{0}^{x} \sum_{n=0}^{\infty}(1-u)^{n} B_{n}^{n} \frac{t^{n}}{n !} d t \\
& =\frac{(1-u) x}{e_{\lambda}(x)-u} \sum_{n=0}^{\infty} \frac{(1-u)^{n} B_{n}^{n}}{n+1} \frac{x^{n}}{n !} \\
= & \left(\sum_{n=0}^{\infty} G_{n, \lambda}^{F}(u) \frac{x^{n}}{n !}\right)\left(\sum_{n=0}^{\infty} \frac{(1-u)^{n} B_{n}^{n}}{n+1} \frac{x^{n}}{n !}\right) \\
\text { L.H.S } & =\sum_{n=0}^{\infty}\left(\sum_{l=0}^{l}\left(\begin{array}{l}
n \\
l
\end{array}\right) \frac{(1-u)^{l} B_{l}^{l}}{l+1} G_{n-l, \lambda}^{F}(u)\right) \frac{x^{n}}{n !} .
\end{aligned}
$$
proof.

By (15) and (16), we require at the desired result. Thus, we complete the

Theorem 2.5. Let $k \geq 1$ and $m \in \mathbb{N} \bigcup\{0\}, s \in \mathbb{C}$, we have

$$
\chi_{k, u, \nu}(-m)=(1-u)^{-m-1}(-1)^{m} G_{m, \nu}^{(F, k)}(u) .
$$

Proof. Let $k \geq 1$, be an integer. For $s \in \mathbb{C}$, we define the function $\chi_{k, \nu}(s)$ as

$$
\chi_{k, \nu}(s)=\frac{1}{\Gamma(s)} \int_{0}^{\infty} \frac{z^{s-1}}{e_{\nu}(z)-u} \operatorname{Ei}_{k}(\log (1+(1-u) z)) d z .
$$

In view of calculation above that $\chi_{k, \nu}(s)$ is holomorphic function for $\Re(s)>0$ because of the comparison test as $\operatorname{Ei}_{k}(\log (1+(1-u) z)) \leq \operatorname{E}_{k}(\log (1+(1-u) z))$ with the assumption $(1-u) t \geq 0$. From (2.18), we note that

$$
\begin{gathered}
\chi_{k, \nu}(s)=\frac{(1-u)^{s-1}}{\Gamma(s)} \int_{0}^{\infty} \frac{z^{s-1}}{e_{\nu}(z)-u} \operatorname{Ei}_{k}(\log (1+(1-u) z)) d z \\
=\frac{(1-u)^{s-1}}{\Gamma(s)} \int_{0}^{1} \frac{z^{s-1}}{e_{\nu}(z)-u} \operatorname{Ei}_{k}(\log (1+(1-u) z)) d z \\
+\frac{(1-u)^{s-1}}{\Gamma(s)} \int_{1}^{\infty} \frac{z^{s-1}}{e_{\nu}(z)-u} \operatorname{Ei}_{k}(\log (1+(1-u) z)) d z
\end{gathered}
$$

The second integral converges absolutely for any $s \in \mathbb{C}$ and hence, the second term on the right hand side vanishes at non-positive integers. That is,

$$
\lim _{s \rightarrow-m}\left|\frac{(1-u)^{s-1}}{\Gamma(s)} \int_{1}^{\infty} \frac{z}{e_{\nu}(z)-u} \operatorname{Ei}_{k}(\log (1+(1-u) z)) d z\right| \leq \frac{(1-u)^{-m-1}}{\Gamma(-m)} M=0
$$

since

$$
\Gamma(s) \Gamma(1-s)=\frac{\pi}{\sin (\pi s)}
$$

On the other hand, for $\Re(s)>0$, the first integral in (2.20) can be written as

$$
\frac{(1-u)^{s-1}}{\Gamma(s)} \int_{0}^{1} \frac{z^{s-1}}{e_{\nu}(z)-u} \operatorname{Ei}_{k}(\log (1+(1-u) z)) d z
$$




$$
\begin{gathered}
=\frac{(1-u)^{s-1}}{\Gamma(s)} \sum_{n=0}^{\infty} \frac{G_{n, \lambda}^{(F, k)}(u)}{n !} \int_{0}^{1} z^{n+s-1} d z \\
=\frac{(1-u)^{s-1}}{\Gamma(s)} \sum_{n=0}^{\infty} \frac{G_{n, \lambda}^{(F, k)}(u)}{n !} \frac{1}{n+s} .
\end{gathered}
$$

which defines an entire function of $s$. Thus, we may include that $\chi_{k, \nu}(s)$ can be continued to an entire function of $s$. Further, from (2.20) and (2.21), we obtain

$$
\begin{gathered}
\chi_{k, \nu, u}(-m)=\lim _{s \rightarrow-m} \frac{(1-u)^{s-1}}{\Gamma(s)} \int_{0}^{1} \frac{z^{s-1}}{e_{\nu}(z)-u} \operatorname{Ei}_{k}(\log (1+(1-u) z)) d z \\
=\lim _{s \rightarrow-m} \frac{(1-u)^{s-1}}{\Gamma(s)} \sum_{r=0}^{\infty} \frac{G_{r, \nu}^{(F, k)}}{s+r} \frac{1}{r !} \\
=\cdots+0+\cdots+0+\lim _{s \rightarrow-m} \frac{(1-u)^{s-1}}{\Gamma(s)} \frac{1}{s+m} \frac{G_{m, \nu}^{(F, k)}(u)}{m !}+0+0+\cdots \\
=\lim _{s \rightarrow-m} \frac{(1-u)^{s-1}}{\Gamma(s)} \frac{\Gamma(1-s) \sin \pi s}{\pi} \frac{G_{m, \nu}^{(F, k)}(u)}{m !}=(1-u)^{-m-1} \Gamma(1+m) \cos (\pi m) \frac{G_{m, \nu}^{(F, k)}}{m !} \\
=(1-u)^{-m-1}(-1)^{m} G_{m, \nu}^{(F, k)}(u) .
\end{gathered}
$$

Thus, we complete the proof of this theorem.

Theorem 2.6. For $n \geq 0$, we have

$$
G_{n, \lambda}^{(F, k)}(x+y ; u)=\sum_{m=0}^{n}\left(\begin{array}{c}
n \\
m
\end{array}\right) G_{n-m, \lambda}^{(F, k)}(x ; u)(y)_{m, \lambda} .
$$

Proof. From (2.1), we have

$$
\begin{gathered}
\sum_{n=0}^{\infty} G_{n, \lambda}^{(F, k)}(x+y ; u) \frac{t^{n}}{n !}=\left(\frac{\operatorname{Ei}_{k}(\log (1+(1-u) t))}{e_{\lambda}(t)-u}\right) e_{\lambda}^{x+y}(t) \\
=\left(\sum_{n=0}^{\infty} G_{n, \lambda}^{(F, k)}(x ; u) \frac{t^{n}}{n !}\right)\left(\sum_{m=0}^{\infty}(y)_{m, \lambda} \frac{t^{m}}{m !}\right) \\
=\sum_{n=0}^{\infty}\left(\sum_{m=0}^{n}\left(\begin{array}{c}
n \\
m
\end{array}\right) G_{n-m, \lambda}^{(F, k)}(x ; u)(y)_{m, \lambda}\right) \frac{t^{n}}{n !}
\end{gathered}
$$

By comparing the coefficients of $T^{n}$, we obtain the result (2.23).

Theorem 2.7. For $n \geq 0$, we have

$$
G_{n, \lambda}^{(F, k)}(x+1 ; u)=\sum_{m=0}^{n}\left(\begin{array}{c}
n \\
m
\end{array}\right) G_{n-m, \lambda}^{(F, k)}(x ; u)(1)_{m, \lambda} .
$$

Proof. By (2.1), we observe that

$$
\begin{gathered}
\sum_{n=0}^{\infty}\left[G_{n, \lambda}^{(F, k)}(x+1 ; u)-G_{n, \lambda}^{(F, k)}(x ; u)\right] \frac{t^{n}}{n !}=\left(\frac{\operatorname{Ei}_{k}(\log (1+(1-u) t))}{e_{\lambda}(t)-u}\right) e_{\lambda}^{x}(t)\left[e_{\lambda}(t)-1\right] \\
=\sum_{n=0}^{\infty} \sum_{m=0}^{n}\left(\begin{array}{c}
n \\
m
\end{array}\right) G_{n-m, \lambda}^{(F, k)}(x ; u)(1)_{m, \lambda} \frac{t^{n}}{n !}-\sum_{n=0}^{\infty} G_{n, \lambda}^{(F, k)}(x ; u) \frac{t^{n}}{n !} .
\end{gathered}
$$

Comparing the coefficients of $t^{n}$ on both sides, we get the result (2.25). 
Theorem 2.8. For $n \geq 0$, we have

$$
G_{n, \lambda}^{(F, k)}(x ; u)=\sum_{m=0}^{n} \sum_{q=0}^{m}\left(\begin{array}{c}
n \\
m
\end{array}\right)(x)_{q} S_{\lambda}^{(2)}(m, q) G_{n-m, \lambda}^{(F, k)}(u) .
$$

Proof. From (2.1), we have

$$
\begin{gathered}
\sum_{n=0}^{\infty} G_{n, \lambda}^{(F, k)}(x ; u) \frac{t^{n}}{n !}=\left(\frac{\operatorname{Ei}_{k}(\log (1+(1-u) t))}{e_{\lambda}(t)-u}\right) e_{\lambda}^{x}(t) \\
=\left(\frac{\operatorname{Ei}_{k}(\log (1+(1-u) t))}{e_{\lambda}(t)-u}\right)\left[e_{\lambda}(t)-1+1\right]^{x} \\
=\left(\frac{\operatorname{Ei}_{k}(\log (1+(1-u) t))}{e_{\lambda}(t)-u}\right)\left(\sum_{q=0}^{\infty}(x)_{q} \sum_{l=q}^{\infty} S_{\lambda}^{(2)}(l, q) \frac{t^{l}}{l !}\right) \\
=\sum_{n=0}^{\infty}\left(\sum_{m=0}^{n} \sum_{q=0}^{m}\left(\begin{array}{c}
n \\
m
\end{array}\right)(x)_{q} S_{\lambda}^{(2)}(m, q) G_{n-m, \lambda}^{(F, k)}(u)\right) \frac{t^{n}}{n !} .
\end{gathered}
$$

By (2.1) and (2.19), we obtain (2.27).

Theorem 2.9. For $n \geq 0$, we have

$$
G_{n, \lambda}^{(F, k)}(x+\alpha \mid u)=\sum_{l=0}^{n} \sum_{m=0}^{l}\left(\begin{array}{c}
n \\
l
\end{array}\right) x^{m} m ! S_{2, \lambda}(l+\alpha, m+\alpha) F_{n-l, \lambda}^{(F, k)}(u) .
$$

Proof. Replacing $x$ by $x+\alpha$ in (2.1), we have

$$
\begin{aligned}
& \sum_{n=0}^{\infty} G_{n, \lambda}^{(F, k)}(x+\alpha ; u) \frac{t^{n}}{n !}=\left(\frac{\operatorname{Ei}_{k}(\log (1+(1-u) t))}{e_{\lambda}(t)-u}\right) e_{\lambda}^{x+\alpha}(t) \\
= & \left(\frac{\operatorname{Ei}_{k}(\log (1+(1-u) t))}{e_{\lambda}(t)-u}\right) e_{\lambda}^{\alpha}(t)\left(\sum_{m=0}^{\infty} x^{m}\left(e_{\lambda}(t)-1\right)^{m}\right) \\
= & \left(\frac{\operatorname{Ei}_{k}(\log (1+(1-u) t))}{e_{\lambda}(t)-u}\right)\left(e_{\lambda}^{\alpha}(t) \sum_{m=0}^{\infty} x^{m} m ! \frac{\left(e_{\lambda}(t)-1\right)^{m}}{m !}\right) \\
= & \left(\frac{\operatorname{Ei}_{k}(\log (1+(1-u) t))}{e_{\lambda}(t)-u}\right)\left(e_{\lambda}^{\alpha}(t) \sum_{m=0}^{\infty} x^{m} m ! \sum_{l=m}^{\infty} S_{2, \lambda}(l, m) \frac{t^{l}}{l !}\right) \\
= & \sum_{n=0}^{\infty} G_{n, \lambda}^{(F, k)}(u) \frac{t^{n}}{n !}\left(\sum_{l=0}^{\infty} \sum_{m=0}^{l} x^{m} m ! S_{2, \lambda}(l+\alpha, m+\alpha) \frac{t^{l}}{l !}\right) \\
\text { L.H.S }= & \sum_{n=0}^{\infty}\left(\sum_{l=0}^{n} \sum_{m=0}^{l}\left(\begin{array}{c}
n \\
l
\end{array}\right) x^{m} m ! S_{2, \lambda}(l+\alpha, m+\alpha) G_{n-l, \lambda}^{(F, k)}(u)\right) \frac{t^{n}}{n !} .
\end{aligned}
$$

Therefore, by (2.1) and (2.30), we get the result (2.29).

\section{Type 2 degenerate unipoly-Frobenius-Genocchi polynomials}

Let $p$ be any arithmetic function which is a real or complex valued function defined on the set of positive integers $\mathbb{N}$. Kim-Kim [7] defined the unipoly function attached to polynomials $p(x)$ by

$$
u_{k}(x \mid p)=\sum_{n=1}^{\infty} \frac{p(n)}{n^{k}} x^{n},(k \in \mathbb{Z}) .
$$


Moreover,

$$
u_{k}(x \mid 1)=\sum_{n=1}^{\infty} \frac{x^{n}}{n^{k}}=\operatorname{Li}_{k}(x),(\text { see }[3]),
$$

is the ordinary polylogaritm function.

By using (3.1), we define the type 2 degenerate unipoly-Frobenius-Genocchi polynomials by

$$
\frac{u_{k}(\log (1+(1-u) t) \mid p)}{e_{\lambda}(t)-u} e_{\lambda}^{x}(t)=\sum_{n=0}^{\infty} G_{n, \lambda, p}^{(F, k)}(x ; u) \frac{t^{n}}{n !} .
$$

In the case when $x=0, G_{n, \lambda, p}^{(F, k)}(u)=G_{n, \lambda, p}^{(F, k)}(0 ; u)$ are called the type 2 degenerate unipoly-Frobenius-Genocchi numbers. Let us take $p(n)=\frac{1}{\Gamma(n)}$. Then, we have

$$
\begin{gathered}
\sum_{n=0}^{\infty} G_{n, \lambda, \frac{1}{\Gamma}}^{(F, k)}(x ; u) \frac{t^{n}}{n !}=\frac{u_{k}\left(\log (1+(1-u) t) \mid \frac{1}{\Gamma} p\right)}{e_{\lambda}(t)-u} e_{\lambda}^{x}(t) \\
=\frac{1}{e_{\lambda}(t)-u} e_{\lambda}^{x}(t) \sum_{m=1}^{\infty} \frac{(\log (1+(1-u) t))^{m}}{m^{k}(m+1) !} \\
=\frac{\operatorname{Ei}_{\mathrm{k}}(\log (1+(1-u) t))}{e_{\lambda}(t)-u} e_{\lambda}^{x}(t) \\
=\sum_{n=0}^{\infty} G_{n, \lambda}^{(F, k)}(x ; u) \frac{t^{n}}{n !}
\end{gathered}
$$

Thus, we have

$$
G_{n, \lambda, \frac{1}{\Gamma}}^{(F, k)}(x ; u)=G_{n, \lambda}^{(F, k)}(x ; u) .
$$

Theorem 3.1. Let $n \in \mathbb{N}$ and $k \in \mathbb{Z}$. Then we have

$$
G_{n, \lambda, p}^{(F, k)}(u)=\sum_{l=0}^{n} \sum_{m=0}^{l}\left(\begin{array}{l}
n \\
l
\end{array}\right) \frac{p(m+1)(m+1) !}{(m+1)^{k}} \frac{S_{1}(l+1, m+1)}{l+1}(1-u)^{l} G_{n-l, \lambda}^{F}(u) .
$$

In particular,

$$
G_{n, \lambda, \frac{1}{\Gamma}}^{(F, k)}(u)=\sum_{l=0}^{n} \sum_{m=0}^{l}\left(\begin{array}{l}
n \\
l
\end{array}\right) \frac{m+1}{(m+1)^{k}} \frac{S_{1}(l+1, m+1)}{l+1}(1-u)^{l} G_{n-l, \lambda}^{F}(u) .
$$

Proof. From (3.3), we get

$$
\begin{gathered}
\sum_{n=0}^{\infty} G_{n, \lambda, p}^{(F, k)}(u) \frac{t^{n}}{n !}=\frac{u_{k}(\log (1+(1-u) t) \mid p)}{e_{\lambda}(t)-u} \\
=\frac{1}{e_{\lambda}(t)-u} \sum_{m=1}^{\infty} \frac{p(m)}{m^{k}}(\log (1+(1-u) t))^{m} \\
=\frac{1}{e_{\lambda}(t)-u} \sum_{m=0}^{\infty} \frac{p(m+1)(m+1) !}{(m+1)^{k}} \sum_{l=m+1}^{\infty} S_{1}(l, m+1) \frac{(1-u)^{l} t^{l}}{l !} \\
=\frac{(1-u) t}{e_{\lambda}(t)-u} \sum_{m=0}^{\infty} \frac{p(m+1)(m+1) !}{(m+1)^{k}} \sum_{m=l}^{\infty} \frac{S_{1}(l+1, m+1)}{l+1}(1-u)^{l} \frac{t^{l}}{l !}
\end{gathered}
$$




$$
\begin{aligned}
& =\left(\sum_{n=0}^{\infty} G_{n, \lambda}^{F}(u) \frac{t^{n}}{n !}\right)\left(\sum_{m=0}^{\infty} \frac{p(m+1)(m+1) !}{(m+1)^{k}} \sum_{m=l}^{\infty} \frac{S_{1}(l+1, m+1)}{l+1}(1-u)^{l} \frac{l^{l}}{l !}\right) \\
& =\sum_{n=0}^{\infty}\left(\sum_{l=0}^{n} \sum_{m=0}^{l}\left(\begin{array}{c}
n \\
l
\end{array}\right) \frac{p(m+1)(m+1) !}{(m+1)^{k}} \frac{S_{1}(l+1, m+1)}{l+1}(1-u)^{l} G_{n-l, \lambda}^{F}(u)\right) \frac{t^{n}}{n !} .
\end{aligned}
$$

Therefore, by comparing the coefficients on both sides of (3.7), we obtain the following theorem.

Theorem 3.2. Let $n \geq 0$ and $k \in \mathbb{Z}$. Then we have

$$
G_{n, \lambda, p}^{(F, k)}(x ; u)=\sum_{m=0}^{n} \sum_{q=0}^{m}\left(\begin{array}{c}
n \\
m
\end{array}\right)(x)_{q} S_{\lambda}^{(2)}(m, q) G_{n-m, \lambda, p}^{(F, k)}(u) .
$$

Proof. Using (3.3), we observe that

$$
\begin{gathered}
\sum_{n=0}^{\infty} G_{n, \lambda, p}^{(F, k)}(x ; u) \frac{t^{n}}{n !}=\left(\frac{u_{k}(\log (1+(1-u) t))}{e_{\lambda}(t)-u}\right) e_{\lambda}^{x}(t) \\
=\left(\frac{u_{k}(\log (1+(1-u) t))}{e_{\lambda}(t)-u}\right)\left[e_{\lambda}(t)-1+1\right]^{x} \\
=\left(\frac{u_{k}(\log (1+(1-u) t))}{e_{\lambda}(t)-u}\right)\left(\sum_{q=0}^{\infty}(x)_{q} \sum_{l=q}^{\infty} S_{\lambda}^{(2)}(l, q) \frac{t^{l}}{l !}\right) \\
\text { L.H.S }=\sum_{n=0}^{\infty}\left(\sum_{m=0}^{n} \sum_{q=0}^{m}\left(\begin{array}{c}
n \\
m
\end{array}\right)(x)_{q} S_{\lambda}^{(2)}(m, q) G_{n-m, \lambda, p}^{(F, k)}(u)\right) \frac{t^{n}}{n !} .
\end{gathered}
$$

By comparing the coefficients of $t^{n}$, we obtain the result (3.8).

Theorem 3.3. Let $n \geq 0$ and $k \in \mathbb{Z}$. Then we have

$$
G_{n, \lambda, p}^{(F, k)}(x ; u)=\sum_{m=0}^{n}\left(\begin{array}{c}
n \\
m
\end{array}\right) G_{n-m, \lambda, p}^{(F, k)}(u)(x)_{m, \lambda} .
$$

Proof. From (3.3), we observe that

$$
\begin{gathered}
\sum_{n=0}^{\infty} G_{n, \lambda, p}^{(F, k)}(x ; u) \frac{t^{n}}{n !}=\frac{u_{k}(\log (1+(1-u) t))}{e_{\lambda}(t)-u} e_{\lambda}^{x}(t) \\
=\left(\sum_{n=0}^{\infty} G_{n, \lambda, p}^{(F, k)}(u) \frac{t^{n}}{n !}\right)\left(\sum_{m=0}^{\infty}(x)_{m, \lambda} \frac{t^{m}}{m !}\right) \\
\text { L.H.S }=\sum_{n=0}^{\infty}\left(\sum_{m=0}^{n}\left(\begin{array}{c}
n \\
m
\end{array}\right) G_{n-m, \lambda, p}^{(F, k)}(u)(x)_{m, \lambda}\right) \frac{t^{n}}{n !} .
\end{gathered}
$$

By comparing coefficients on both sides of (3.11), we obtain the following theorem. 


\section{Conclusions}

Motivated by the definition of the type 2 degenerate poly-Bernoulli polynomials introduced by Kim et al. [18], in the present paper, we have considered a class of new generating function for the Frobenius-Genocchi polynomials, called the type 2 degenerate poly-Frobenius-Genocchi polynomials, by means of the polyexponential function. Then, we have derived some useful relations and properties. We have showed that the type 2 degenerate poly-Frobenius-Genocchi polynomials equal a linear combination of the degenerate Frobenius-Genocchi polynomials and degenerate Stirlings numbers of the first and second kind. In a special case, we have given a relation between the type 2 degenerate Frobenius-Genocchi polynomials and Bernoulli polynomials of order $n$. Moreover, inspired by the definition of unipoly-Bernoulli polynomials introduced by Kim-Kim [7] we have introduced the type 2 degenerate unipolyFrobenius-Genocchi polynomials by means of unipoly function and given multifarious properties including degenerate Stirling numbers of the second kind and degenerate Frobenius-Genocchi polynomials.

Author Contributions: All authors contributed equally to the manuscript and typed, read, and approved final manuscript.

Conflict of Interest: The authors declare no conflict of interest.

Funding: None.

Acknowledgements: None.

\section{References}

[1] Carlitz, L. Degenerate Stirling, Bernoulli and Eulerian numbers. Utilitas Math. 1979, 15, 51-88.

[2] Carlitz, L. A degenerate Staudt-Clausen theorem, Arch. Math. (Basel) 1956, 7, 28-33.

[3] Kaneko, M. "poly-Bernoulli numbers". J. Théor Nombres Bordeaux. 1997, 9(1), 221-228.

[4] Khan, W.A.; Srivastava, D. On the generalized Apostol type FrobeniusGenocchi polynomials. Filomat. 2019, 33(7), 1967-1977.

[5] Khan, W.A.; Ahmad, M. Partially degenerate poly-Bernoulli polynomials. Adv. Stud. Contemp. Math., 2018, 28(3), 487-496.

[6] Khan, W.A. A new class of degenerate Frobenius-Euler Hermite polynomials. Adv. Stud. Contemp. Math., 2018, 30(4), 567-576.

[7] Kim, D.S.; Kim, T. A note on polyexponential and unipoly functions. Russ. J. Math. Phys. bf 2019, 26(1), 40-49.

[8] Kim, T.; Kim, D.S. An identity of symmetry for the degenerate FrobeniusEuler polynomials. Math. Slovaca. 2018, 68(1), 239-243.

[9] Kim, T.; Kwon, H.I.; Seo, J.J. On the degenerate Frobenius-Euler polynomials. arXiv:1507.04846v1 [math. N.T] 17 July 2015.

[10] Kim, D.S.; Kim, T.; Seo, J.J. Higher-order Bernoulli, Frobenius-Euler and Euler polynomials, J. Comput. Anal. Appl. 2014, 17(1), 147-155.

[11] Kim, T. A note on degenerate Stirling polynomials of the second kind. Proc. Jangjeon Math. Soc. 2017, 20(3), 319-331. 
[12] Kim, T.; Kim, D.S. Degenerate polyexponential functions and degenerate Bell polynomials. J. Math. Anal. Appl. 2020, 487(2), 124017.

[13] Kim, T.; Kim, D.S. A note on central Bell numbers and polynomials. Russ. J. Math. Phys. 2020, 27(1), 76-81.

[14] Kim, D.S.; Kim, T. A note on a new type of degenerate Bernoulli numbers. Russ. J. Math. Phys. 2020, 27(2), 227-235.

[15] Kim, T.; Kim, D.S.; Kim, H.Y.; Kwon, J. Degenerate Stirling polynomials of the second kind and some applications. Symmetry. 2019, 11(8), Article ID 1046.

[16] Kim, T.; Kim, D.S. Degenerate Laplace transform and degenerate gamma function. Russ. J. Math. Phys. 2017, 24(2), 241-248.

[17] Kim, T.; Kim, D.S.; Dolgy, D.V.; Kwon, J. Some identities on degenerate Genocchi and Euler numbers. Informatica. 2020, 31(4), 42-51.

[18] T. Kim, D. S. Kim, H. Y. Kim, L.-C, Jang, Degenerate poly-Bernoulli numbers and polynomials, Informatica. 3(13) (2020), 2-8.

[19] Yasar, B.Y.; Ozraslan, M.A. Frobenius-Euler and Frobenius-Genocchi polynomials and their different equations.New trens in Mathematical Sciences. 2015, 3(2), 172-180. 